\title{
TWISTED HIGGS BUNDLES AND THE FUNDAMENTAL GROUP OF COMPACT KÄHLER MANIFOLDS
}

\author{
O. García-Prada And S. Ramanan
}

\begin{abstract}
We study polystable Higgs bundles twisted by a line bundle over a compact Kähler manifold. These form a Tannakian category when the first and second Chern classes of the bundle are zero. In this paper we identify the corresponding Tannaka group in the case in which the line bundle is of finite order. This group is described in terms of the pro-reductive completion of the fundamental group of the manifold, and the character associated to the line bundle.
\end{abstract}

\section{Introduction}

Let $(X, \omega)$ be a compact Kähler manifold. A Higgs bundle over $X$ consists of a holomorphic vector bundle $E \rightarrow X$ together with a sheaf homomorphism $\varphi: E \rightarrow E \otimes \Omega^{1}$, satisfying $\varphi \wedge \varphi=0$, where $\Omega^{1}$ is the holomorphic cotangent bundle of $X$. A notion of stability for Higgs bundles, similar to that of vector bundles, was first introduced for a Riemann surface by Hitchin in [H1], where he proved that this is a necessary and sufficient condition for the existence of an irreducible solution to the so-called self-duality equations for for a Hermitian metric on $E$. His results were later extended to the general case by Simpson [Si1]. Of special interest is the moduli space of stable Higgs bundles $E$ for which

$$
\mathrm{c}_{1}(E) \cdot[\omega]^{\operatorname{dim} X-1}=0 \text { and } \operatorname{ch}_{2}(E) \cdot[\omega]^{\operatorname{dim} X-2}=0,
$$

where $\mathrm{c}_{1}(E)$ and $\mathrm{c}_{2}(E)$ are the first and second Chern classes of $E$, and $\operatorname{ch}_{2}(E)=$ $1 / 2 \mathrm{c}_{1}(E)^{2}-\mathrm{c}_{2}(E)$. The moduli space of such Higgs bundles can be identified with the moduli space of irreducible flat complex connections, which in turn is in correspondence with the moduli of complex irreducible representations of the fundamental group of $X$. This is proved by using, on one hand, Hitchin's and Simpson's existence theorem and, on the other, a theorem of Donaldson [D2] and Corlette $[\mathrm{C}]$ on the existence of harmonic metrics on flat bundles.

In this paper we shall deal with a twisted version of Higgs bundles. Let $L$ be a holomorphic line bundle over $X$. An L-twisted Higgs bundle over $X$ (or just twisted if there is no confusion), is a pair consisting of a holomorphic vector bundle $E$ and a sheaf morphism $\theta: E \rightarrow E \otimes L \otimes \Omega^{1}$, i.e. an element $\theta \in H^{0}\left(\right.$ End $\left.E \otimes L \otimes \Omega^{1}\right)$, satisfying $\theta \wedge \theta=0$. Stability is defined exactly in

Received August 13, 1999.

Key words and phrases. Higgs bundle, stability, representation of the fundamental group, flat connection, Tannakian category. 
the same way as for ordinary Higgs bundles. There are many interesting aspects of Higgs bundles that are shared by the twisted theory: twisted Higgs bundles, like ordinary Higgs bundles, have nice moduli spaces $[\mathrm{N}, \mathrm{Y}]$, and define also, under certain conditions, complete integrable systems in a generalised sense [H2, Bo, Ma]. There is one aspect, however, that to our knowledge has not yet been studied, namely, their relation to the fundamental group. The main indication that a certain relationship has to exist comes from the fact that polystable $L$-twisted Higgs bundles, with the vanishing condition on the first and second Chern characters as above, like Higgs bundles, form a Tannakian category [Si2], which, by the Tannaka duality theorem, is dual to a certain pro-reductive group, from the representations of which the category can be recovered. In the ordinary Higgs bundle theory the group is the pro-reductive completion of the fundamental group of $X-$ as one deduces from the theorems of Hitchin, Simpson, Donaldson, Corlette mentioned above. The problem we wish to address is to find the corresponding group in the twisted situation. In this paper, we take the first steps in this direction by considering the case in which the $\operatorname{deg} L=0$ and $L$ is of finite order, i.e. $L^{n}$ is isomorphic to the trivial line bundle for some $n$. In this situation $L$ corresponds to a unitary character $\pi_{1}(X) \rightarrow \mathrm{U}(1)$ of the fundamental group of $X$, and we can give a description of the group in terms of the pro-reductive completion of $\pi_{1}(X)$ - the Tannaka group for the untwisted category — and this character.

\section{Twisted Higgs bundles}

1.1. Twisted Higgs bundles and Hermitian metrics. Let $(X, \omega)$ be a compact Kähler manifold and let $L$ be a holomorphic line bundle over $X$. An $L$ twisted Higgs bundle over $X$ is a pair $(E, \theta)$ consisting of a holomorphic vector bundle $E$ over $X$ and a Higgs field $\theta \in H^{0}\left(\right.$ End $\left.E \otimes L \otimes \Omega^{1}\right)$, satisfying $\theta \wedge \theta=0$, where $\Omega^{1}$ is the holomorphic cotangent bundle of $X$. A twisted Higgs bundle $(E, \theta)$ is said to be stable if and only if $\mu\left(E^{\prime}\right)<\mu(E)$ for every proper coherent subsheaf $E^{\prime} \subset E$ invariant under $\theta$, i.e. $\theta\left(E^{\prime}\right) \subset E^{\prime} \otimes \Omega^{1}$. Recall that the slope of a $E^{\prime}$ is defined as $\mu\left(E^{\prime}\right)=\operatorname{deg}\left(E^{\prime}\right) / \operatorname{rank}\left(E^{\prime}\right)$, where $\operatorname{deg}\left(E^{\prime}\right)=c_{1}\left(E^{\prime}\right) \cdot[\omega]^{\operatorname{dim} X-1}$.

The notion of stability is related to the existence of a special Hermitian metric on $E$. More precisely:

Theorem 1. [Li] Let $(E, \theta)$ be an L-twisted Higgs bundle. Let us fix a Hermitian metric on L. The existence of a Hermitian metric $h$ on $E$ satisfying

$$
\Lambda F_{h}+\Lambda\left[\theta, \theta^{*}\right]=\lambda I,
$$

is equivalent to the polystability of $(E, \theta)$.

Here $F_{h}$ is the curvature of the unique connection compatible with the Hermitian metric as well as the holomorphic structure on $E$, and $\Lambda$ is the contraction with the Kähler form. The constant $\lambda$ is determined by the slope of $E$, and $I$ is the identity endomorphism of $E$. By $\theta^{*}$ we denote the adjoint of $\theta$ with respect 
to $h$ and the metric of $L$, and $\left[\theta, \theta^{*}\right]=\theta \theta^{*}+\theta^{*} \theta$ is the usual extension of the Lie bracket to forms with values in the algebra of endomorphisms. By polystability we mean that $(E, \theta)$ is a direct sum of twisted Higgs bundles of the same slope as $E$ (zero in this case).

When $L$ is the trivial line bundle one has the ordinary Higgs bundles theory studied by Hitchin [H1] on Riemann surfaces and Simpson [Si1] in the higher dimensional case. When $\theta=0,(1)$ reduces to the Hermitian-Einstein equation and one obtains the theorem of Narasimhan and Seshadri, Donaldson, and Uhlenbeck and Yau [AB, D1, D3, D4, NS, UY].

Equation (1) has, as in the untwisted case, a symplectic interpretation. It corresponds to the moment map for the action of the unitary group on the product Kähler manifold $\mathcal{A} \times \Omega^{1,0}(E n d E \otimes L)$, where $\mathcal{A}$ is the space of unitary connections on the $C^{\infty}$ Hermitian vector bundle $(E, h)$. The moduli space of stable $L$-twisted Higgs bundles is then obtained as a Kähler quotient inheriting in this way a Kähler structure. A construction of the moduli of $L$-twisted Higgs bundles using Geometric Invariant Theory has been given by Nitsure [N] for Riemann surfaces and Yokogawa $[\mathrm{Y}]$ in higher dimensions.

1.2. Twisted Higgs bundles and Tannakian categories. Twisted Higgs bundles can be regarded from the point of view of Tannakian categories. This is the point of view taken by Simpson [Si2] in his study of ordinary Higgs bundles, and we will follow his approach. (See also [De, DMOS, Sa, T] for more details about Tannakian categories). A tensor category is a category $\mathcal{C}$ with a functorial binary operation $\otimes: \mathcal{C} \times \mathcal{C} \rightarrow \mathcal{C}$. An associative and commutative tensor category is a tensor category provided with additional natural isomorphisms expressing associativity and commutativity of the tensor product that have to satisfy certain canonical axioms. A unit 1 is an object 1 provided with natural isomorphisms $1 \otimes V \cong V$ satisfying canonical axioms. A functor $\mathcal{F}$ between associative and commutative categories with unit is a functor provided with natural isomorphisms $\mathcal{F}(U \otimes V) \cong \mathcal{F}(U) \otimes \mathcal{F}(V)$. A neutral Tannakian category $\mathcal{C}$ is an associative and commutative tensor category with unit, which is abelian, rigid (duals exist), $\operatorname{End}(1)=\mathbf{C}$, and which is provided with an exact, faithful fibre functor $\mathcal{F}: \mathcal{C} \rightarrow$ Vect, where Vect is the tensor category of complex, finite dimensional vector spaces.

If $G$ is an affine group scheme over $\mathbf{C}$ the category $\operatorname{Rep}(G)$ of complex representations of $G$ is a neutral Tannakian category. The fibre functor $\mathcal{F}_{G}$ is given by by sending a representation of $G$ to the underlying vector space. The group $G$ is recovered as the group $G=\operatorname{Aut}^{\otimes}\left(\mathcal{F}_{G}\right)$ of tensor automorphisms of the fibre functor. The converse is given by the fundamental duality theorem of Tannaka-Grothendieck-Saavedra ([De, DMOS, Sa]).

Theorem 1.1. Let $(\mathcal{C}, \mathcal{F})$ be a neutral Tannakian category and let $G=$ $\operatorname{Aut}^{\otimes}(\mathcal{F})$ be the group of tensor automorphisms of the fibre funtor. Then $(\mathcal{C}, \mathcal{F}) \cong\left(\operatorname{Rep}(G), \mathcal{F}_{G}\right)$. 
We shall briefly describe the group $\operatorname{Aut}^{\otimes}(\mathcal{F})$ to which we shall refer sometimes as the Tannaka group of the Tannakian category $(\mathcal{C}, \mathcal{F})$ (see [Si2] and the references mentioned above for a detailed account.) Let $\operatorname{End}(\mathcal{F})$ be the algebra of endomorphisms of the the fibre functor. Its elements are collections $\left\{f_{V}\right\}$ with $f_{V} \in \operatorname{End}(\mathcal{F}(V))$ such that for any morphism $\psi: V \rightarrow W$, one has $\mathcal{F}(\psi) f_{V}=f_{W} \mathcal{F}(\psi)$. Let $\operatorname{Aut}^{\otimes}(\mathcal{F})$ be the set of elements $\left\{f_{V}\right\}$ of $\operatorname{End}(\mathcal{F})$ satisfying

$$
f_{1}=1 \quad f_{V \otimes W}=f_{V} \otimes f_{W} .
$$

The existence of duals in $\mathcal{C}$ implies that any element in $\operatorname{Aut}^{\otimes}(\mathcal{F})$ consists entirely of automorphisms, and hence there is no need to include a condition for invertibility. The algebra $\operatorname{End}(\mathcal{F})$ is a projective limit of finite dimensional algebras and it is endowed with a projective limit topology. The subset $\operatorname{Aut}^{\otimes}(\mathcal{F})$ has a structure of projective limit of algebraic varieties.

Let $G$ be a group such that $\operatorname{Rep}(G)$, with the functor $\mathcal{F}_{G}$ defined as usual, is a Tannakian category. There is a map form $G$ to $\operatorname{Aut}^{\otimes}\left(\mathcal{F}_{G}\right)$ which sends an element $g \in G$ to the natural automorphims $\left\{f_{V}\right\}$ of $\mathcal{F}_{G}$ defined by setting $f_{V}$ equal to the action of $g$ on the vector space $\mathcal{F}_{G}(V)$ underlying the representation $V$. As mentioned above, for complex affine group schemes this map is an isomorphism.

We come now to the Tannakian nature of twisted Higgs bundles.

Proposition 1.2. The tensor category of polystable L-twisted Higgs bundles $E$ over $X$, satisfying $\mathrm{c}_{1}(E) \cdot[\omega]^{\operatorname{dim} X-1}=0$ and $\operatorname{ch}_{2}(E) \cdot[\omega]^{\operatorname{dim} X-2}=0$, with fibre functor defined by sending an L-twisted Higgs bundle to the fibre of the bundle at a fixed point of $X$, is a neutral Tannakian category.

Proof. Let $(E, \theta)$ and $(F, \eta)$ be two $L$-twisted Higgs bundles. Its tensor product is given by the $L$-twisted Higgs bundle $(E \otimes F, \theta \otimes 1+1 \otimes \eta)$. The polystability of the tensor product can be proved directly, but it follows also from the existence of metrics satisfying (1). The tensor product of the two metrics satisfies (1) as well and hence the tensor product Higgs bundle is polystable by Theorem 1 . This operation defines an associative and commutative tensor category. Suppose $f:(E, \theta) \rightarrow(F, \eta)$ is a morphism of $L$-twisted Higgs bundles, namely a sheaf homomorphism $f: E \rightarrow F$ such that the appropriate diagram commutes. Then the subsheaf $V$ of $F$ generated by the image is invariant under $\eta$, and the kernel of $f$ is invariant under $\theta$, since their generic fibres are clearly invariant. Now we have $\operatorname{deg} E / \operatorname{ker} f \geq 0$ since $E$ is semistable and $\operatorname{deg} V \leq 0$ since $F$ is semistable. But then we have a generic isomorphism induced by $f$ from $E / \operatorname{ker} f \rightarrow V$. This is only possible if the degrees of both these bundles are 0 , and the above map is actually an isomorphism. This shows that semistable $L$-twisted Higgs bundles of degree 0 , form an abelian category. The dual of a pair $(E, \theta)$ is the pair $\left(E^{*}, \theta^{\vee}\right)$, where $E^{*}$ is the dual bundle to $E$ and $\theta^{\vee}$ is the map obtained by transposing $\theta$ and tensoring with the canonical line bundle. Obviously, the Higgs bundle $(\mathcal{O}, 0)$ is a unit and satisfies that $\operatorname{End}((\mathcal{O}, 0))=\mathbf{C}$. 
The fibre functor $\mathcal{F}$ is defined by choosing a point $x \in X$ and sending $(E, \theta)$ to the fibre of $E$ at the point $x$. The faithfulness of the $\mathcal{F}$ follows from the polystability of $(E, \theta)$.

In this paper we shall address the problem of describing the corresponding Tannaka group when $\operatorname{deg} L=0$. We will give an answer to this problem when the order of $L$ is finite, that is when $L^{n}$ is the trivial line bundle for some $n$. This answer is given in terms of the Tannaka group of the category of ordinary Higgs bundles, i.e. those for which $L$ is the trivial line bundle.

\section{Ordinary Higgs bundles}

Our goal in this section is to describe the Tannaka group of the category of polystable (untwisted) Higgs bundles $E$ a compact Kähler manifold $(X, \omega)$ satisfying

$$
c_{1}(E) \cdot[\omega]^{\operatorname{dim} X-1}=0 \text { and } \operatorname{ch}_{2}(E) \cdot[\omega]^{\operatorname{dim} X-2}=0 .
$$

This is done by means of the correspondence between polystable Higgs bundles satisfying (2) and semisimple complex representations of the fundamental group. In the sequel we briefly recall the main ideas of this correspondence (see [Si1, H1, D2, C] for details).

2.1. Higgs bundles, flat bundles and representations of the fundamental group. To associate a complex representation to a Higgs bundle we will pass through the intermediate category of flat bundles. Let $V$ be $C^{\infty}$ complex vector bundle of rank $r$ over $X$, and let $D$ be a $\operatorname{GL}(r, \mathbf{C})$ connection on $V$. We say that $D$ is flat if its curvature vanishes, i.e. $D^{2}=0$. If $D$ is a flat connection on $V$ the pair $(V, D)$ is called a flat bundle since, by using the flat connection, one can find an open cover of $X$ with constant transition functions for $V$. If $D$ is a connection on a vector bundle $V$ and $x$ is a (fixed) point of $X$ recall that the holonomy group of $D$ is the group of endomorphisms of $V_{x}$ obtained by parallel transport along all closed curves starting at $x$. If $D$ is flat the parallel displacement depends only on the homotopy class of the closed curve and defines a homomorphism

$$
\rho: \pi_{1}(X, x) \longrightarrow \mathrm{GL}\left(V_{x}\right)
$$

whose image is the holonomy of $D$. Conversely, given a representation $\rho$ : $\pi_{1}(X, x) \rightarrow \mathrm{GL}(r, \mathbf{C})$ one can construct a vector bundle $V$ of rank $r$ with a flat connection by setting

$$
V=\tilde{X} \times{ }_{\rho} \mathbf{C}^{r}
$$

where $\tilde{X}$ is the universal cover of $X$ and $\tilde{X} \times{ }_{\rho} \mathbf{C}^{r}$ is the quotient of $\tilde{X} \times \mathbf{C}^{r}$ by the action of $\pi_{1}(X, x)$ given by $(y, v) \mapsto(\gamma(y), \rho(\gamma) v)$ for $\gamma \in \pi_{1}(X, x)$ (regarded as the covering transformation group acting on $\tilde{X})$. The trivial connection on $\tilde{X} \times \mathbf{C}^{r}$ descends to give a flat connection on $V$, whose holonomy is the image of $\rho$. 
The relation between flat bundles and Higgs bundles involves a certain class of metrics over a flat bundle - the so-called harmonic metrics. Let $(V, D)$ be a flat bundle over $X$. Given a metric $h$ on $V$ we can decompose $D$ uniquely as $D=\nabla+\Psi$ where $\nabla$ is a unitary connection on $V$ and $\Psi$ is a is a 1 -form with values in the self-adjoint endomorphisms of $V$. The metric $h$ is said to be harmonic if

$$
\nabla^{*} \Psi=0
$$

where we use the metric on $X$ to define $\nabla^{*}$. A metric $h$ on $V$ is just a section of a certain $\operatorname{GL}(r, \mathbf{C}) / U(r)$-bundle over $X$. This can be viewed as a $\pi_{1}(X)$ equivariant function

$$
\tilde{h}: \tilde{X} \rightarrow \mathrm{GL}(r, \mathbf{C}) / U(r),
$$

where $\tilde{X}$ is the universal cover of $X$. It turns out that $\nabla^{*} \Psi=0$ is equivalent to the condition that the map $\tilde{h}$ should be harmonic. In fact the one-form $\Psi$ can be identified with the differential of $\tilde{h}$, and $\nabla$ with the pull-back of the Levi-Civita connection on $\mathrm{GL}(r, \mathbf{C}) / U(r)$.

To state an existence theorem for such metrics we need the following definitions. A flat bundle $(V, D)$ is said to be irreducible if $V$ has no non-trivial $D$-invariant subbundles. It will be called semisimple if any $D$-invariant subbundle has a $D$-invariant complement. Any semisimple connection is a direct sum of irreducible ones.

Theorem 2.1. A flat bundle $(V, D)$ over $X$ admits a harmonic metric if and only if it is semisimple.

This theorem is proved by Donaldson [D2] for rank 2 bundles when $X$ is a Riemann surface, and in full generality (including the base manifold being a compact Riemannian manifold of arbitrary dimension) by Corlette [C].

Let $(E, \varphi)$ be a Higgs bundle over $X$. We want to associate to it a flat bundle over $X$. This is not always possible, but if $(E, \varphi)$ supports a hermitian metric $h$ satisfying $\Lambda F_{h}+\Lambda\left[\varphi, \varphi^{*}\right]=0$ then we can consider the pair $(V, D)$, taking $V$ to be the underlying $C^{\infty}$ bundle to $E$ and $D=\bar{\partial}_{E}+\partial_{h}+\varphi+\varphi^{*}$, where $\partial_{h}$ is a differential operator such that $\bar{\partial}_{E}+\partial_{h}$ is the unique connection compatible with the metric and the holomorphic structure of $E$. A simple computation shows the following.

Lemma 2.2. $\bar{\partial}_{E} \varphi=0, \varphi \wedge \varphi=0$ and $F_{h}+\left[\varphi, \varphi^{*}\right]=0$ imply that $D$ is flat, i.e. $D^{2}=0$.

We will show now how to associate a Higgs bundle to a flat bundle. Let $(V, D)$ be a flat bundle over $X$. We want to produce out of it a stable Higgs bundle $(E, \varphi)$ over $X$. Let $h$ be a Hermitian metric on $V$. We can decompose $D$ in its $(1,0)$ and $(0,1)$ componentes

$$
D=D^{\prime}+D^{\prime \prime}
$$


and consider the unique operators $D_{h}^{\prime \prime}$ and $D_{h}^{\prime}$ so that $D^{\prime}+D_{h}^{\prime \prime}$ and $D_{h}^{\prime}+D^{\prime \prime}$ become $h$-unitary connections. Let

$$
\partial_{h}=\frac{D^{\prime}+D_{h}^{\prime}}{2}, \quad \bar{\partial}_{h}=\frac{D^{\prime \prime}+D_{h}^{\prime \prime}}{2}, \quad \varphi_{h}=\frac{D^{\prime}-D_{h}^{\prime}}{2}, \quad \varphi_{h}^{*}=\frac{D^{\prime \prime}-D_{h}^{\prime \prime}}{2} .
$$

It is not difficult to see that

Lemma 2.3. $D^{2}=0$ implies that $\varphi_{h} \wedge \varphi_{h}=0$ and $F_{h}+\left[\varphi_{h}, \varphi_{h}^{*}\right]=0$. Where $F_{h}$ is the curvature of $\bar{\partial}_{h}+\partial_{h}$.

Of course $\bar{\partial}_{h}$ defines a holomorphic structure on $V$, but $\varphi_{h}$ need not be holomorphic with respect to it, i.e. there is no reason why $\bar{\partial}_{h} \varphi_{h}=0$. This happens precisely when the metric is harmonic ([D2, H1, Si2]).

Putting everything together one has the following.

Theorem 2.4. There is a tensor functor which is an equivalence of categories between the category of polystable Higgs bundles over $(X, \omega)$ satisfying (2) and the category of semisimple flat bundles which, in turn, is equivalent to the tensor category of semisimple complex representations of the fundamental group of $X$.

2.2. The Tannaka group for ordinary Higgs bundles. One can easily prove the following [Si2, Lemma 6.1].

Proposition 2.5. Let $H$ be a finitely generated group. The tensor category of semisimple representations of $H$, with its obvious fibre functor, is a neutral Tannakian category whose Tannaka group is naturally isomorphic to the proreductive completion of $H$.

We recall that the pro-reductive completion of a group $H$ is a projective limit $G=\lim (\Gamma, \rho)$, where the inverse limit runs over the directed system of representations $\rho: H \rightarrow \Gamma$ for complex reductive groups $\Gamma$ (we shall assume that the image of $\rho$ is Zariski dense for convenience). An arrow $(\Gamma, \rho) \rightarrow\left(\Gamma^{\prime}, \rho^{\prime}\right)$ consists of a homomorphism $f: \Gamma \rightarrow \Gamma^{\prime}$ such that $f \rho=\rho^{\prime}$. The group $G$ is characterised by the following universal property: For every representation $H \rightarrow \Gamma$ into a complex reductive group there exists a unique extension $G \rightarrow \Gamma$ such that the following diagram

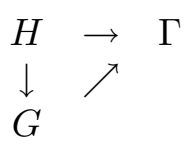

commutes.

From this proposition and Theorem 2.4 one concludes the following.

Theorem 2.6. Let $G$ be the Tannaka group of the category of polystable Higgs bundles over $X$ satisfying (2). Then $G$ is naturally isomorphic to the proreductive completion of $\pi_{1}(X, x)$. 
One of the main ingredients for the description of the Tannaka group in the twisted situation is a natural action of the group $\mathbf{C}^{*}$ on the category of polystable Higgs bundles given by

$$
(E, \varphi) \mapsto(E, \lambda \varphi) \text { for every } \lambda \in \mathbf{C}^{*} .
$$

This action induces an action of $\mathbf{C}^{*}$ on the category of semisimple representations of the fundamental group. It should be pointed out that, while this action is very clear and explicit from the point of view of Higgs bundles, its explicit effect on a representation of the fundamental group is not easy to describe.

One can formalise the action of $\mathbf{C}^{*}$ on a Tannakian category $(\mathcal{C}, \mathcal{F})$ in terms of certain tensor functors satisfying canonical axioms. If the action preserves the fibre functor $\mathcal{F}$ one has an action of $\mathbf{C}^{*}$ on $\operatorname{End}(\mathcal{F})$ by sending the element $\left\{f_{V}\right\}$ of $\operatorname{End}(\mathcal{F})$ to $\left\{f_{V}^{\lambda}\right\}$ with $f_{V}^{\lambda}=f_{\lambda V}$ for every $\lambda \in \mathbf{C}^{*}$, and hence one has an action on the Tannaka group $\operatorname{Aut}^{\otimes}(\mathcal{F})$. The action of $\mathbf{C}^{*}$ on the category of polystable pairs preserves clearly the fibre functor since the bundle is unchanged, and one can then transfer this action to $G$ - the pro-reductive completion of the fundamental group. More precisely one has the following theorem ([Si2][Theorem $6])$.

Theorem 2.7. There exists a unique action of $\mathbf{C}^{*}$ on $G$, each $\lambda \in \mathbf{C}^{*}$ acting by a homomorphism of pro-reductive groups, such that if $\rho: G \rightarrow \operatorname{GL}(n, \mathbf{C})$ is the representation corresponding to $(E, \varphi)$, then $\rho \circ \lambda$ is the representation corresponding to $(E, \lambda \varphi)$.

\section{Main theorem}

Before stating our main result we will prove some preliminary necessary facts on groups with a $\mathbf{C}^{*}$-action.

3.1. Twisted groups. Let $G$ be a group and let $\mathbf{C}^{*}$ act on $G$, i.e. we have a homomorphism

$$
\mathbf{C}^{*} \longrightarrow \operatorname{Aut}(G)
$$

For every $\lambda \in \mathbf{C}^{*}$ and $g \in G$ we shall denote by $g^{\lambda}$ the image of $g$ by the automorphism $G \rightarrow G$ defined by $\lambda$.

Proposition 3.1. Let $\chi: G \rightarrow \mathbf{C}^{*}$ be a character satisfying

$$
\chi(g)=\chi\left(g^{\lambda}\right) \text { for every } g \in G, \lambda \in \mathbf{C}^{*} .
$$

We can define a group $G_{\chi}$ by taking the underlying set to be $G$ and the group operation to be

$$
g * h=g h^{\chi^{-1}(g)} \quad \text { for every } g, h \in G .
$$


Proof. Associativity results from the following computation for $g, h, k \in G$.

$$
\begin{aligned}
g *(h * k)= & g(h * k)^{\chi^{-1}(g)} \\
& =g\left(h k^{\chi^{-1}(h)}\right)^{-1}(g) \\
& =g h^{\chi^{-1}(g)} k^{\chi^{-1}(h) \chi^{-1}(g)} \\
& =g h^{\chi^{-1}(g)} k^{\chi^{-1}(g h)} . \\
(g * h) * k=g h^{\chi^{-1}(g)} k^{\chi^{-1}\left(g h^{\chi^{-1}(g)}\right)} & \left.g h^{\chi^{-1}(g)} k^{\chi^{-1}(g) \chi^{-1}\left(h^{-1}(g)\right.}\right) \\
= & g h^{\chi^{-1}(g)} k^{\chi^{-1}(g) \chi^{-1}(h)} \text { by condition }(3) \\
= & g h^{\chi^{-1}(g)} k^{\chi^{-1}(g h)} .
\end{aligned}
$$

The identity element $e \in G$ is also the identity element of $G_{\chi}$. Indeed,

$$
g * e=g e^{\chi^{-1}(g)}=g e=g .
$$

Let $g \in G$. Take $h$ to be the preimage of $g^{-1}$ under the automorphism $\chi^{-1}(g)$ : $G \rightarrow G$, i.e. $h^{\chi^{-1}(g)}=g^{-1}$. Then

$$
g * h=g h^{\chi^{-1}(g)}=g g^{-1}=e
$$

and hence $h$ is the inverse of $g$. We have then proved that $G_{\chi}=(G, *)$ is a group.

We shall now prove few properties about $G_{\chi}$ that will be useful later.

Proposition 3.2. Let $\chi: G \rightarrow \mathbf{C}^{*}$ be a character. Then the map $\alpha_{\chi}: G_{\chi} \rightarrow \mathbf{C}^{*}$ defined by $\alpha_{\chi}(g)=\chi(g)$ is a character of $G_{\chi}$. In particular $K=\operatorname{ker} \chi$ is a normal subgroup of $G_{\chi}$.

Proof.

$$
\begin{aligned}
\alpha_{\chi}(g * h) & =\chi\left(g h^{\chi^{-1}(g)}\right) \\
& =\chi(g) \chi\left(h^{\chi^{-1}(g)}\right) \\
& =\chi(g) \chi(h) \text { by }(3) \\
& =\alpha_{\chi}(g) \alpha_{\chi}(h) \text { for every } g, h \in G_{\chi}
\end{aligned}
$$

Proposition 3.3. The action of $\mathbf{C}^{*}$ on $G$ defines an action of $\mathbf{C}^{*}$ on $G_{\chi}$. Moreover, this action leaves $K$ invariant. 
Proof.

$$
\begin{aligned}
(g * h)^{\lambda} & =\left(g h^{\chi^{-1}(g)}\right)^{\lambda} \\
& =g^{\lambda} h^{\chi^{-1}(g) \lambda} \\
& =g^{\lambda} h^{\lambda \chi^{-1}(g)} \\
& =g^{\lambda} h^{\lambda \chi^{-1}\left(g^{\lambda}\right)} \text { by }(3) \\
& =g^{\lambda} * h^{\lambda} .
\end{aligned}
$$

To see that the action of $\mathbf{C}^{*}$ on $G_{\chi}$ leaves $K$ invariant, let $g \in K$, and $\lambda \in \mathbf{C}^{*}$. By (3), we see that

$$
\alpha_{\chi}\left(g^{\lambda}\right)=\chi\left(g^{\lambda}\right)=\chi(g)=e
$$

and hence $g^{\lambda} \in K$.

3.2. Main theorem. Let $L$ be a holomorphic line bundle over $X$ such that $\operatorname{deg} L=0$. Let $\pi_{1}(X)=\pi_{1}(X, x)$ be the fundamental group of $X$ with respect to a fixed point $x \in X$. The line bundle $L$ corresponds to a unitary character $\chi^{\prime}: \pi_{1}(X) \rightarrow \mathrm{U}(1)$. That is, if $\tilde{X}$ is the universal cover of $X, L$ is the line bundle associated to the $\pi_{1}(X)$-principal bundle $\tilde{X} \rightarrow X$ via the representation $\chi^{\prime}$.

Let $G$ be the pro-reductive completion of $\pi_{1}(X)$. As discussed in section 1.2, $G$ is isomorphic to the Tannaka group of the category of polystable Higgs bundles and there is $\mathbf{C}^{*}$ on $G$, induced from the action of $\lambda \in \mathbf{C}^{*}$ on a Higgs bundle $(E, \varphi)$ given by $(E, \varphi) \mapsto(E, \lambda \varphi)$ (Theorem 2.7). We shall apply the construction of a twisted group structure given above to the pro-reductive completion of the fundamental group of $X$.

Proposition 3.4. Let $\chi: G \rightarrow \mathbf{C}^{*}$ be the extension to $G$ of a unitary character $\chi^{\prime}: \pi_{1}(X) \rightarrow \mathrm{U}(1)$. Then the action of $\mathbf{C}^{*}$ on $G$ considered above satisfies (3), i.e.

$$
\chi(g)=\chi\left(g^{\lambda}\right) \text { for every } g \in G, \lambda \in \mathbf{C}^{*} .
$$

Proof. We shall regard $G$ as the group of tensor automorphisms of the fibre functor of the Tannakian category of Higgs bundles over $X$ (see section 1.2). Hence $g \in G$ basically associates to any stable Higgs pair $(E, \varphi)$ an automorphism $f_{(E, \varphi)}$ of the fibre $E_{x}$, in a functorial way. Let $\lambda \in \mathbf{C}^{*}$. The element $g^{\lambda} \in G$ associates to $(E, \varphi)$ the automorphism $f_{(E, \lambda \varphi)}$.

Let now $\rho$ be a semisimple representation of $G$. Of course $\rho$ gives rise to a semisimple representation of $\pi_{1}(X, x)$ and hence to a polystable Higgs bundle $(E, \varphi)$. One can see that

$$
\rho(g)=f_{(E, \varphi)} .
$$

But if $\left.\rho\right|_{\pi_{1}(X)}$ is unitary, then the associated Higgs bundle is $(E, 0)$, and hence $\rho(g)=\rho\left(g^{\lambda}\right)$. In particular, for our "unitary" character we have $\chi(g)=\chi\left(g^{\lambda}\right)$, which concludes the proof.

We can thus consider the group $G_{\chi}$ by means of the construction given in the previous section. 
We are now ready to state the main theorem of this paper.

Theorem 3.5. Let $L$ be a holomorphic line bundle of degree zero and finite order. Then $G_{\chi}$ is the Tannaka group of the category of polystable L-twisted Higgs bundles satisfying (2). More precisely, there is a tensor functor which is an equivalence of categories between the category of polystable L-twisted Higgs bundles satisfying (2) and the category of semisimple complex representations of $G_{\chi}$.

It is natural to conjecture that this result is also true in the infinite order case. We hope to come back to this in a future paper.

\section{Proof of main theorem}

Let $\chi^{\prime}: \pi_{1}(X) \rightarrow \mathrm{U}(1)$ be the unitary character corresponding to $L$ and let $\Gamma=\operatorname{Im} \chi^{\prime}$ be the image. Let $\tilde{X}$ be the universal cover of $X$ and let $Y=$ $\tilde{X} / \operatorname{Ker} \chi^{\prime}$. Then $\operatorname{Ker} \chi^{\prime} \cong \pi_{1}(Y)$ and one has the covering map

$$
p: Y \longrightarrow X
$$

whose Galois group is $\Gamma=\operatorname{Im} \chi^{\prime} \cong \pi_{1}(X) / \pi_{1}(Y)$.

The basic strategy that we shall follow in our approach is to translate our problem into a problem on $Y$ : If the line bundle $L$ is of finite order, i.e. if a finite power of $L$ is isomorphic to the trivial line bundle, $\Gamma$ is a finite cyclic group and hence $Y$ is compact. The pull-back of $L$ to $Y$ is of course trivial, and we can thus use the untwisted theory over $Y$ to prove our theorem.

4.1. Twisted Higgs bundles and representations of $\pi_{1}(Y)$. Let $(E, \theta)$ be an $L$-twisted Higgs bundle over $X$. Its pull-back to $Y,(F, \varphi)=\left(p^{*} E, p^{*} \theta\right)$, becomes a genuine Higgs bundle. It is not difficult to characterize Higgs bundles on $Y$ that come from twisted Higgs bundles on $X$.

Proposition 4.1. There is an equivalence of tensor categories between the category of L-twisted Higgs bundles $(E, \theta)$ on $X$ and the category of Higgs bundles $(F, \varphi)$ on $Y$ that satisfy

$$
\gamma^{*} F=F \quad \text { and } \gamma^{*} \varphi=\gamma \varphi \text { for every } \gamma \in \Gamma .
$$

Proof. We must first clarify our notation: On the one hand we regard $\Gamma$ as the Galois group of the cover $Y \rightarrow X$, and on the other as a subgroup of $\mathrm{U}(1)$. So when we write $\gamma^{*} \varphi$ we are thinking of $\gamma$ as a transformation of $Y$, while when we write $\gamma \varphi$ we regard $\gamma$ as an element of $U(1)$ that multiplies the Higgs field.

One direction is clear: If $(E, \theta)$ is an $L$-twisted Higgs bundle over $X$ its pullback, $(F, \varphi)=\left(p^{*} E, p^{*} \theta\right)$, obviously satisfies (5). To see the converse, observe that the first condition in (5) amounts to saying that the bundle $F \rightarrow Y$ descends to a bundle $E \rightarrow X$, since the Galois group is cyclic. It is obvious that this correspondence is compatible with tensor products.

Proposition 4.2. Let $(E, \theta)$ be an L-twisted Higgs bundle on $X$, and let $(F, \varphi)$ its pull-back to $Y$. The pair $(E, \theta)$ is polystable if and only if $(F, \varphi)$ is polystable. 
Proof. The vector bundle $F=p^{*} E$ is a $\Gamma$-equivariant vector bundle, and one can consider for the pair $(F, \varphi)$ a weaker stability notion consisting of the usual stability condition, but only for $\Gamma$-equivariant subsheaves of $F$. One can show $[G]$ that this $\Gamma$-equivariant condition for polystability is equivalent to the polystability of $(F, \varphi)$. Now, suppose that $(E, \theta)$ is not stable. The pull-back of the destabilizing subsheaf would violate the $\Gamma$-equivariant stability of $(F, \varphi)$. Conversely, if $(F, \varphi)$ is not $\Gamma$-equivariantly stable, then let $F^{\prime} \subset F$ be a $\Gamma$-equivariant $\varphi$-invariant destabilizing subbundle. Because of $\Gamma$-equivariance $F^{\prime}$ descends to a $\theta$-invariant destabilizing subbundle of $E$.

Another way of proving this proposition is to use Theorem 1: If $(F, \varphi)$ is polystable there exists a Hermitian metric on $F$ solving equation (1). By the uniqueness of the solution, this metric must be $\Gamma$-invariant and hence descend to a metric on $E$ solving (1) implying the polystability of $(E, \theta)$. The converse is also clear.

Since, as we know, there is a tensor functor which is an equivalence of categories between the category of polystable Higgs bundles over $Y$ and the category of semisimple representations of the fundamental group of $Y$, we shall now give an interpretation of condition (5) in terms of representations of $\pi_{1}(Y)$. To do this we need to understand the two actions of $\Gamma$ on Higgs bundles in terms of the representations of $\pi_{1}(Y)$. To explain the action corresponding to pull-backing by $\gamma \in \Gamma$, we shall digress a little.

Let $G$ be a group and let $K \subset G$ be a normal subgroup. Let $\Gamma=G / K$. We have a short exact sequence

$$
1 \longrightarrow K \longrightarrow G \longrightarrow \Gamma \longrightarrow 1 \text {. }
$$

The group $G$ acts on the set of representations of $K$, via inner automorphisms, i.e. if $\rho$ is a representaion of $K, g \in G$ sends $\rho$ to $\left.\rho \circ \operatorname{Int}_{g}\right|_{K}$, where

$$
\left.\operatorname{Int}_{g}\right|_{K}(h)=g h g^{-1} \text { for every } h \in K \text {. }
$$

Proposition 4.3. Let $\gamma \in \Gamma$ and let $g_{\gamma} \in G$ be a lift of $\gamma$. The map

$$
[\rho] \mapsto \gamma \cdot[\rho]=\left[\left.\rho \circ \operatorname{Int}_{g_{\gamma}}\right|_{K}\right] \quad \text { for every }[\rho] \in \operatorname{Rep}(K) \text { and } \gamma \in \Gamma
$$

defines an action of $\Gamma$ on the set $\operatorname{Rep}(K)$ of equivalence classes of representations of $K$.

Proof. It is clear since two lifts of $\gamma$ differ by an element of $K$.

The following is immediate.

Proposition 4.4. Let $\rho$ be a representation of $K$ and let $\gamma \in \Gamma$. Then $\gamma \cdot[\rho]=$ $[\rho]$ for every $\gamma \in \Gamma$ is equivalent to $[\rho]=\left[\rho \circ \operatorname{Int}_{g}\right]$ for every $g \in G$.

In our situation we have the extension

$$
1 \longrightarrow \pi_{1}(Y) \longrightarrow \pi_{1}(X) \longrightarrow \Gamma \longrightarrow 1 \text {. }
$$


By the Proposition 4.3 there is an action of $\Gamma$ on representations of $\pi_{1}(Y)$, given for every $[\rho] \in \operatorname{Rep}\left(\pi_{1}(Y)\right)$ and $\gamma \in \Gamma$ by $[\rho] \mapsto \gamma \cdot[\rho]=\left[\rho \circ \operatorname{Int}_{g_{\gamma}}\right]$, where $g_{\gamma} \in$ is any lift of $\gamma$ to $\pi_{1}(X)$. The following is clear.

Proposition 4.5. The action of $\Gamma$ on $\operatorname{Rep}\left(\pi_{1}(Y)\right)$ given above corresponds to the action of $\Gamma$ on the set of equivalence classes of Higgs bundles and flat bundles over $Y$ defined by $\gamma \cdot(F, \varphi)=\left(\gamma^{*} F, \gamma^{*} \varphi\right)$ and $\gamma \cdot(V, D)=\left(\gamma^{*} V, \gamma^{*} D\right)$, respectively.

As we saw in section 1.2 , the action of $\mathbf{C}^{*}$ on the moduli of stable Higgs bundles on $Y$ given for $\lambda \in \mathbf{C}^{*}$, by $[(F, \varphi)]^{\lambda}=[(F, \lambda \varphi)]$ defines an action on $\operatorname{Rep}\left(\pi_{1}(Y)\right)$. If $[\rho]$ corresponds to $[(F, \varphi)]$ we denote by $[\rho]^{\lambda}$ the representation corresponding to $[(F, \lambda \varphi)]$. The following proposition follows immediately.

Proposition 4.6. Let $(F, \varphi)$ be a polystable Higgs bundle over $Y$, and let $[\rho]$ be the corresponding semisimple representation of $\pi_{1}(Y)$. The condition (5) is equivalent to

$$
\gamma \cdot[\rho]=[\rho]^{\gamma} \text { for every } \gamma \in \Gamma .
$$

We would like now to relate the representations of $\pi_{1}(Y)$ satisfying (7) to representations of $G_{\chi}$. To do this we will relate them first to the representations of $K=\operatorname{Ker} \chi$.

\subsection{From representations of $\pi_{1}(Y)$ to representations of $K$.}

Proposition 4.7. If $\chi^{\prime}: \pi_{1}(X) \rightarrow \mathrm{U}(1)$ be a unitary character of finite order of $\pi_{1}(X)$. Let $\chi$ be its extension to $G$, the Tannaka closure of $\pi_{1}(X)$, and let $K=\operatorname{Ker} \chi$. Then any complex representation of $\pi_{1}(Y)$ extends to a complex representation of $K$.

Proof. Let $n$ be the order of $\chi^{\prime}$. Since $\chi^{\prime n}$ is trivial, we get from the uniqueness of extensions that $\chi^{n}$ is also trivial. Hence we have the diagram

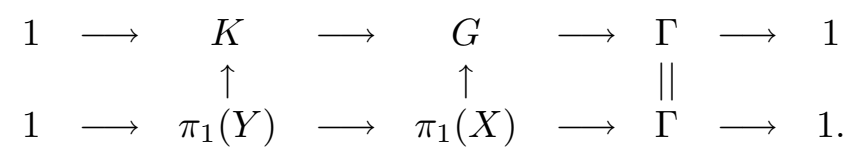

Let $(V, \rho)$ be an irreducible representation of $\pi_{1}(Y)$. This induces a semisimple representation $(W, \operatorname{ind}(\rho))$ of $\pi_{1}(X)$, where

$W=\left\{f: \pi_{1}(X) \rightarrow V \mid f(h y)=\rho\left(y^{-1}\right) f(h)\right.$, for every $\left.y \in \pi_{1}(Y), h \in \pi_{1}(X)\right\}$.

The morphism $\operatorname{ind}(\rho) \in \operatorname{Aut}(W)$ is defined by

$$
(h f)(x)=\operatorname{ind}(\rho)_{h}(f)(x)=f\left(h^{-1} x\right) \text { for every } h, x \in \pi_{1}(X) \text { and } f \in W .
$$

This representation extends of course to a representation of $G$ since $G$ is the Tannaka closure of $\pi_{1}(X)$. Let $\mathcal{F}(G)$ be the set of polynomial functions on $G$ with values in $V$, and let $e: W \rightarrow V$ be the evaluation map $f \mapsto f(1)$. Consider the map $\Phi: W \rightarrow \mathcal{F}(G)$, defined by

$$
\Phi(w)(g)=e\left(g^{-1} w\right) .
$$


Let us also consider the restriction map Res : $\mathcal{F}(G) \rightarrow \mathcal{F}\left(\pi_{1}(X)\right)$, where $\mathcal{F}\left(\pi_{1}(X)\right)$ is the set of functions on $\pi_{1}(X)$. The image of Res is contained in $W$ and the composite $W \stackrel{\Phi}{\longrightarrow} \mathcal{F}(G) \stackrel{\text { Res }}{\longrightarrow} W$ is the identity since $\Phi(w)(h)=$ $e\left(h^{-1} w\right)=\left(h^{-1} w\right)(1)=w(h)$, that is $\left.\Phi(w)\right|_{\pi_{1}(X)}=w$.

The affine group $G$ is defined as Spec of the algebra of representation functions (namely coefficients of semisimple representations) of $\pi_{1}(X)$. So the restriction map of the algebra of functions on $G$ to the Zariski closure of $\pi_{1}(X)$ is onto (because it is affine) and one-to-one, and hence they are the same. In other words $\pi_{1}(X)$ is dense in $G$. Now, the image of $W$ in $\mathcal{F}(G)$ satisfies

$$
f(g y)=\rho\left(y^{-1}\right) f(h), \text { for every } y \in \pi_{1}(Y), g \in G .
$$

This is because $\psi(g)=f(g y)-\rho\left(y^{-1}\right) f(g)$ is 0 on $\pi_{1}(X)$ and the image of $\pi_{1}(X)$ is Zariski dense on $G$. Let

$$
W^{\prime}=\left\{f \in \mathcal{F}(G) \mid f(g y)=\rho\left(y^{-1}\right) f(g), \text { for every } y \in \overline{\pi_{1}(Y)}, g \in G\right\} .
$$

The restriction map $W^{\prime} \rightarrow \mathcal{F}\left(\pi_{1}(X)\right)$ is injective and the image is $W$, therefore $W^{\prime}=W$ and hence $G$ acts on it and the dimension of $W$ must be $\left|G / \overline{\pi_{1}(Y)}\right| \operatorname{dim} V$ which has to coincide with $|G / K| \operatorname{dim} V$. Hence $\overline{\pi_{1}(Y)}=K$.

We shall now show that $K$ acts on Ker $e$ and hence on $V$. Let $f, f^{\prime} \in W$. From $\left(f-f^{\prime}\right)(1)=0$ we have $\left(f-f^{\prime}\right)(y)=0$ for every $y \in \pi_{1}(Y)$ and since $\overline{\pi_{1}(Y)}=K$ we have $\left(f-f^{\prime}\right)(k)=\rho(k)\left(f-f^{\prime}\right)(1)=0$, which complets the proof.

Corollary 4.8. $K$ is the Tannaka closure of $\pi_{1}(Y)$.

We thus conclude that if $[\rho]$ is a representation of $\pi_{1}(Y)$ satisfying (7) it extends to a representation of $K$ satisfying the same condition, where the first action of $\Gamma$ on this representation is defined via the extension

$$
1 \longrightarrow K \longrightarrow G \longrightarrow \Gamma \longrightarrow 1,
$$

and (6). On the other hand, since $K$ is the Tannaka closure of $\pi_{1}(Y)$, by Theorem 2.7 , there is an action of $\mathbf{C}^{*}$ on $K$ such that

$$
[\rho]^{\lambda}=[\rho \circ \lambda] \text { for every } \lambda \in \mathbf{C}^{*} .
$$

Recall that we are identifying $\lambda \in \mathbf{C}^{*}$ with the homomorphism $K \rightarrow K$ that it defines.

Combining all this with the results of the previous subsection, we have the following.

Proposition 4.9. There is a functor which is an equivalence of categories between the category of polystable L-twisted Higgs bundles over $X$ and the category of semisimple representations $[\rho]$ of $K$ satisfying

$$
\gamma \cdot[\rho]=[\rho]^{\gamma} \text { for every } \gamma \in \Gamma .
$$


From section 3.1, we know that $K$ is also a subgroup of $G_{\chi}$. We shall show now that the representations of $K$ corresponding to twisted Higgs bundles extend to representations of $G_{\chi}$. To see this we shall briefly analyse separately the general problem of extending a representation of a normal subgroup to the whole group.

\subsection{Extending representations of a normal subgroup.}

Proposition 4.10. Let $G$ be a group and $K \subset G$ be a normal subgroup. Let $(V, \rho)$ be a representation of $K$ which extends to a representation of $G$, then for every $g \in G$, the representations $\rho$ and $\rho \circ \operatorname{Int}_{g}$ are equivalent.

Proof. Suppose that $(V, \rho)$ extends to a representation $(V, \tilde{\rho})$ of $G$. Then for every $g \in G$ and $h \in K$ we have

$$
\left(\rho \circ \operatorname{Int}_{g}\right)(h)=\rho\left(g h g^{-1}\right)=\tilde{\rho}\left(g h g^{-1}\right)=\tilde{\rho}(g) \tilde{\rho}(h) \tilde{\rho}\left(g^{-1}\right)=\tilde{\rho}(g) \rho(h) \tilde{\rho}\left(g^{-1}\right) .
$$

Thus $\rho$ and $\rho \circ \operatorname{Int}_{g}$ are equivalent.

We are interested in the case in which $\Gamma=G / K$ is a finite cyclic group. In this situation, the converse of the previous Proposition is actually true. More precisely:

Proposition 4.11. Let $K$ be a normal subgroup of $G$ and let $\Gamma=G / K$ be a finite cyclic group. Let $(V, \rho)$ be a semisimple representation of $K$ such that $\gamma \cdot[\rho]=[\rho]$ for every $\gamma \in \Gamma$. Then $(V, \rho)$ extends to a semisimple representation of $G$.

Proof. Assume first that $\rho$ is irreducible. Let $n$ be the order of $\Gamma$ and let $a$ be a generator of $\Gamma$. Let $g \in G$ be a lift of $a$. By assumption there exists a matrix $T \in \mathrm{GL}(V)$ such that

$$
\rho\left(g k g^{-1}\right)=T \rho(k) T^{-1} \quad \text { for every } k \in K .
$$

This implies that $T^{n} \rho(k) T^{-n}=\rho\left(g^{n} k g^{-n}\right)$. But $g^{n} \in K$ and so we have $T^{n} \rho(k) T^{-n}=\rho\left(g^{n}\right) \rho(k) \rho\left(g^{-n}\right)$. In other words, $\rho\left(g^{-n}\right) T^{n}$ commutes with $\rho(k)$ for all $k \in K$. Since $\rho$ is irreducible, $\rho\left(g^{-n}\right) T^{n}=\lambda I$ for some constant $\lambda \in \mathbf{C}$, by Schur's Lemma, and replacing $T$ by $\mu T$ with $\mu^{n}=\lambda$, we see that $T^{n}=\rho\left(g^{n}\right)$. We will now extend $\rho$ to a representation $\tilde{\rho}$ of $G$ by setting $\tilde{\rho}(g)=T$. Since $G$ is defined as the quotient of the free product of $K$ and $\Gamma$ with the relation $g^{n}=k$, for some $k \in K$, we see that $\tilde{\rho}$ is indeed a representation of $G$ extending $\rho$.

Suppose now that $\rho=\bigoplus_{i=1}^{m} \rho_{i}$, where the $\rho_{i}^{\prime} s$ are irreducible, inequivalent representations such that

$$
\rho_{i}=\left.\rho_{1} \circ \operatorname{Int}_{g^{i-1}}\right|_{K} \quad \text { for } \quad 1 \leq i \leq m,
$$

with $m$ dividing $n$. Every representation satisfying the hypothesis of the theorem is a direct sum of representations of this kind, and it will hence be enough to consider this case.

The automorphism $T$ in (9) has a block decomposition of the form 


$$
T=\left(\begin{array}{cccc}
0 & \ldots & 0 & A_{m} \\
A_{1} & 0 & \ldots & 0 \\
\ldots & \ldots & \ldots & \ldots \\
0 & \ldots & A_{m-1} & 0
\end{array}\right)
$$

where $A_{i}$ with $1 \leq i \leq m$ is an invertible linear transformation. In terms of this decomposition (9) is equivalent to

$$
\begin{array}{ccc}
A_{m} \rho_{m}(k) A_{m}^{-1} & = & \rho_{1}\left(g k g^{-1}\right) \\
A_{1} \rho_{1}(k) A_{1}^{-1} & = & \rho_{2}\left(g k g^{-1}\right) \\
\cdots & \ldots & \cdots \\
A_{m-1} \rho_{m-1}(k) A_{m-1}^{-1} & = & \rho_{m}\left(g k g^{-1}\right) .
\end{array}
$$

This implies that

$$
\rho_{1}(k)=A_{m} A_{m-1} \ldots A_{1} \rho_{1}\left(g^{m} k g^{-m}\right) A_{1}^{-1} \ldots A_{m-1}^{-1} A_{m}^{-1},
$$

and similarly for the other $\rho_{i}^{\prime} s$. More precisely, let

$$
B_{i}=\prod_{j=1}^{i-1} A_{i-j} \prod_{l=0}^{m-i} A_{m-l} .
$$

We have

$$
\rho_{i}(k)=B_{i} \rho_{i}\left(g^{m} k g^{-m}\right) B_{i}^{-1} \text { for } 1 \leq i \leq m .
$$

Iterating this, we obtain

$$
\rho_{i}(k)=B_{i}^{p} \rho_{i}\left(g^{n} k g^{-n}\right) B_{i}^{-p} \text { for } 1 \leq i \leq m,
$$

where $p=n / m$. Since $g^{n} \in K$,

$$
\rho_{i}(k)=B_{i}^{p} \rho_{i}\left(g^{n}\right) \rho_{i}(k) \rho_{i}\left(g^{-n}\right) B_{i}^{-p} \text { for } 1 \leq i \leq m,
$$

from which, by Schur's Lemma, we obtain

$$
\rho_{i}\left(g^{n}\right)=\lambda_{i} B_{i}^{p} \text { for } 1 \leq i \leq m
$$

for constants $\lambda_{i} \in \mathbf{C}$. Now, taking $k=g^{n}$ in (11), we get

$$
\operatorname{Tr} \rho_{i}\left(g^{n}\right)=\operatorname{Tr} \rho_{j}\left(g^{n}\right) \text { for } 1 \leq i, j \leq m .
$$

But $\operatorname{Tr}\left(B_{i}^{p}\right)=\operatorname{Tr}\left(B_{j}^{p}\right)$, for $1 \leq i, j \leq m$, since $A_{i} B_{i}^{p} A_{i}^{-1}=B_{i+1}^{p}$, and hence (13) and (14) imply that $\lambda_{i}=\lambda_{j}=\lambda$ for $1 \leq i, j \leq m$. As above, we can replace $T$ by $\mu T$ with $\mu^{n}=\lambda$, so that $T^{n}=\rho\left(g^{n}\right)$, and extend $\rho$ to a representation $\tilde{\rho}$ of $G$ by setting $\tilde{\rho}(g)=T$.

The proof is now complete since, as mentioned above, every representation satisfying the hypothesis of our theorem is a direct sum of representations of the kind defined by (10), and $\mathrm{T}$ can be decomposed in diagonal blocks to which we can apply the above argument. 
4.4. From representations of $K$ to representations of $G_{\chi}$. Coming back to our main theme, recall that $K=\operatorname{ker} \chi$ is a normal subgroup of $G_{\chi}$, and we want to see that the representations of $K$ coming from twisted Higgs bundles extend to representations of $G_{\chi}$. More precisely:

Proposition 4.12. There is a tensor functor which is an equivalence of categories between the category of semisimple representations of $K$ satisfying (8) and the category of semisimple representations of $G_{\chi}$.

Proof. Let us consider the extension

$$
1 \longrightarrow K \longrightarrow G_{\chi} \longrightarrow \Gamma_{\chi} \longrightarrow 1
$$

where $\Gamma_{\chi}=G_{\chi} / K$. By Proposition $4.3 \Gamma_{\chi}$ acts on $\operatorname{Rep}(K)$. Let us denote this action by $\gamma \cdot \chi[\rho]$, for every $[\rho] \in \operatorname{Rep}(K)$ and $\gamma \in \Gamma_{\chi}$. We will show now that (8) is equivalent to

$$
\gamma \cdot \chi[\rho]=[\rho] \text { for every } \gamma \in \Gamma_{\chi},
$$

and hence if (8) is satisfied, since $\Gamma_{\chi}$ is cyclic, we can apply Proposition 4.11 to conclude that a semisimple representation of $K$ extends to a semisimple representation of $G_{\chi}$. To see this, let $\operatorname{Int}_{g}^{\chi}$ the inner automorphism of $G_{\chi}$ defined by $g \in G_{\chi}$. We have to take some care about what we mean by the inner automorphism defined by $g$ since the underlying sets of $G$ and $G_{\chi}$ coincide, but the group structures are different. The restriction of $\operatorname{Int}_{g}^{\chi}$ to $K$ is the homomorphism

$$
\operatorname{Int}_{g}^{\chi}(h)=g * h * g_{\chi}^{-1}, \text { for } g \in G_{\chi} \text { and } h \in K,
$$

where $g_{\chi}^{-1}$ denotes the inverse of $g$ with respect to the operation $*$ defined by (4).

By Proposition 4.4, (15) is equivalent to

$$
[\rho]=\left[\rho \circ \operatorname{Int}_{g}^{\chi}\right] \text { for every } g \in G_{\chi} .
$$

Now, for every $g \in G_{\chi}$ and $h \in K$

$$
\operatorname{Int}_{g}^{\chi}(h)=g * h * g_{\chi}^{-1}=g\left(h\left(g_{\chi}^{-1}\right)^{\chi^{-1}(h)}\right)^{\chi^{-1}(g)}=g h^{\chi^{-1}(g)} g^{-1}
$$

since $\chi^{-1}(h)=1$ and $\left(g_{\chi}^{-1}\right)^{\chi^{-1}(g)}=g^{-1}$. We can thus conclude that $\operatorname{Int}_{g}^{\chi}=$ $\operatorname{Int}_{g} \circ \chi^{-1}(g)$ and hence $(16)$ can be rewritten as $[\rho]=\left[\rho \circ \operatorname{Int}_{g} \circ \chi^{-1}(g)\right]$ or equivalently

$$
[\rho \circ \chi(g)]=\left[\rho \circ \operatorname{Int}_{g}\right]
$$

Hence

$$
\gamma \cdot \chi[\rho]=\left[\rho \circ \operatorname{Int}_{g_{\gamma}}^{\chi}\right]=\left[\rho \circ \operatorname{Int}_{g_{\gamma}} \circ \gamma^{-1}\right]
$$

and (15) is thus equivalent to (8).

We have thus completed the proof of Theorem 3.5. 
Remark. Another way to prove our main theorem could be, perhaps, the following. The twisted Higgs bundle $(E, \theta)$ defines a Higgs bundle $(V, \Theta)$ over $X$ by taking $V=\bigoplus_{i=0}^{n-1} E \otimes L^{i}$, where $n$ is the order of $\Gamma$, and

$$
\Theta=\left(\begin{array}{cccc}
0 & \ldots & 0 & \theta \\
\theta & 0 & \ldots & 0 \\
\ldots & \ldots & \ldots & \ldots \\
0 & \ldots & \theta & 0
\end{array}\right)
$$

If $(E, \theta)$ is stable it should not be difficult to prove that $(V, \Theta)$ is polystable, defining then a representation of $G$. This is the representation induced by the representation of $K$ corresponding to the twisted Higgs bundle $(E, \theta)$. One would need then to characterize these representations of $G$ and show that they are in bijection with the representations of $G_{\chi}$.

Acknowledgements. The research carried out in this paper has taken place during visits of the second author to Universidad Autónoma (Madrid) and of the first author to TIFR (Mumbai) and Ecole Polytechnique (Palaiseau), as well as joint visits to ICTP (Trieste) and the Mathematical Institute (Oxford). We wish to thank these institutions for their hospitality and support. This work has been partly supported by the Spanish MEC PB95-0185. Both authors have benefited from workshops organised by the VBAC research group under the European algebraic networks AGE and Europroj, supported by the European Union.

\section{References}

[AB] M. F. Atiyah and R. Bott, The Yang-Mills equations over Riemann surfaces, Philos. Trans. Roy. Soc. Lond. Ser. A 308 (1982), 523-615.

[Bo] F. Bottacin, Symplectic Geometry on moduli spaces of stable pairs, Ann. Sci. École Norm. Sup. 28 (1995), 391-433.

[C] K. Corlette, Flat G-bundles with canonical metrics, J. Differential Geom. 28 (1988), 361-382.

[De] P. Deligne, Catégories tannakiennes, The Grothendieck Festschrift, Volume II, Progr. Math. 87, Birkhäuser, 1990, pp. 111-195.

[DMOS] P. Deligne, J. S. Milne, A. Ogus, and K. Shih, Hodge Cycles, Motives, and Shimura Varieties, Lecture Notes in Mathematics 900 (1982), Springer-Verlag.

[D1] S. K. Donaldson, A new proof of a theorem of Narasimhan and Seshadri, J. Differential Geom. 18 (1983), 269-277.

[D2] S. K. Donaldson, Twisted harmonic maps and the self-duality equations, Proc. London Math. Soc. 55 (1987), 127-131.

[D3] S. K. Donaldson, Anti-self-dual Yang-Mills connections on a complex algebraic surface and stable vector bundles, Proc. London Math. Soc. 50 (1985), 1-26.

[D4] S. K. Donaldson, Infinite determinants, stable bundles and curvature, Duke Math. J. 54 (1987), 231-247.

[G] O. García-Prada, Invariant connections and vortices, Commun. Math. Phys. 156 (1993), 527-546.

[H1] N. J. Hitchin, The self-duality equations on a Riemann surface, Proc. London Math. Soc. 55 (1987), 59-126.

[H2] N. J. Hitchin, Stable bundles and integrable systems, Duke Math. J. 54 (1987), 91-114. 
[Li] T. R. Lin, Hermitian-Yang-Mills-Higgs metrics and stability for holomorphic vector bundles with Higgs fields over a compact Kähler manifold, Ph.D. Thesis, UC-San Diego (1989).

[Ma] E. Markman, Spectral curves and integrable systems, Compositio Math. 93 (1994), 255-290.

[NS] M. S. Narasimhan and C.S. Seshadri, Stable and unitary bundles on a compact Riemann surface, Ann. Math. 82 (1965), 540-564, Proc. Nat. Acad. Sci. U.S.A. 52 (1964), 207-211.

[N] N. Nitsure, Moduli space of semistable pairs on a curve, Proc. London Math. Soc. 62 (1991), 275-300.

[Sa] N. Saavedra Rivano, Catégories Tannakiennes, Lecture Notes in Mathematics 265, Springer-Verlag, 1972.

[Si1] C.T. Simpson, Constructing variations of Hodge structure using Yang-Mills theory and applications to uniformization, J. Amer. Math. Soc. 1 (1988), 867-918.

[Si2] C. T. Simpson, Higgs bundles and local systems, Inst. Hautes Études Sci. Publ. Math. 75 (1992), 5-95.

[T] T. Tannaka, Über den Dualitätssatz der nichtkommutativen topologischen Gruppen, Tohoku Math. J. 45 (1938), 1-12.

[UY] K. K. Uhlenbeck and S. T. Yau, On the existence of Hermitian-Yang-Mills connections on stable bundles over compact Kähler manifolds, Comm. Pure Appl. Math. 39-S (1986), S257-S293.

[Y] K. Yokogawa, Moduli of stable pairs, J. Math. Kyoto Univ. 31 (1991), 311-327.

Departamento de Matemáticas, Universidad Autónoma de Madrid, 28049 Madrid, SPAIN.

E-mail address: oscar.garcia-prada@uam.es

School of Mathematics, Tata Institute of Fundamental Research, Homi Bhabha Road, Mumbai - 400 005, India.

E-mail address: ramanan@math.tifr.res.in 